\title{
Multi-Objective Optimisation using Optimizer WSS to Support Operation and Planning Decisions of Melbourne Water Supply System
}

\author{
$\underline{\text { M.D.U.P Kularathna }}^{\text {a }}$, T. S. C. Rowan ${ }^{\text {b }}$, H. Schultz-Byard ${ }^{\text {b }}$, D. R. Broad ${ }^{\text {b }}$, D. McIver ${ }^{\text {b }}$, D. Flower ${ }^{\text {a }}$, \\ B. Baker ${ }^{\text {a }}$, B. G. Rhodes ${ }^{a}$ and P. J. Smith ${ }^{b}$ \\ ${ }^{\mathrm{a}}$ Melbourne Water, Melbourne, Victoria, Australia, ${ }^{\mathrm{b}}$ Optimatics, Adelaide, South Australia, Australia \\ Email: udaya.kularathna@melbournewater.com.au
}

\begin{abstract}
In the last decade, many communities in Australia have faced prolonged drought periods. Water authorities have responded with various demand and supply measures ranging from water conservation and water restrictions to seawater desalination. Most of the above measures are associated with some environmental, economic and/or social impacts. These measures can also affect the key objectives of water utilities such as providing a reliable supply of low cost, high quality water while maintaining the environmental health. Some of the above objectives tend to be mutually conflicting, highlighting that decisions associated with key operations and system planning must be based on an assessment of such multiple objectives.
\end{abstract}

For many water authorities, the multi-objective decisions on key operations and system planning need to be made considering uncertainties in future streamflow conditions. For water supply systems that have the capacity to store large volumes of water, this may require the consideration of potential streamflow conditions over many future years. In addition, the decisions may also involve trading-off short-term operations such as greater use of higher-cost water sources with longer-term solutions to supply-demand balance. The latter may include a range of demand management or supply augmentation measures. The complexity of making the above decisions calls for a multi-objective optimisation approach that considers key operation decisions and supply-demand balance decisions over temporal and spatial scales ranging from long-term strategic decisions down to more detailed annual system operation planning. Despite this clear need, optimisation tools are not widely available or used in the water industry. In contrast, simulation modelling tools, such as REALM, have been widely used by many water authorities in Australia to assist with system planning and operations. REALM simulates the water allocation within a system in accordance with user-defined operating rules, but does not optimise the system operations over time.

The integration of new supplies and the need to consider multiple objectives in water system planning and operations prompted Melbourne Water to engage water system optimisation specialists Optimatics to develop a decision support system, Optimizer WSS, for water supply system optimisation. Optimizer WSS employs a Multi-Objective Genetic Algorithm (MOGA) optimisation which in turn uses Melbourne Water's existing REALM simulation model to efficiently assess the consequences of millions of combinations of alternative decisions on key operations and the long-term supply-demand balance for a range of potential future hydrological scenarios. The optimisation identifies superior solutions termed 'Pareto-Optimal solutions' considering the range of water supply objectives. The Pareto-Optimal solutions enable Melbourne Water and its stakeholders to select the most suitable solution by optimally balancing one or more water supply objectives against the others, through a Multi-Criteria Decision Analysis (MCDA) considering an array of additional assessment criteria as well. The complex issue of streamflow uncertainty is addressed through a streamflow-scenario based optimisation followed by testing of optimal solutions for their robustness under different streamflow scenarios. The whole process is built on a high level of stakeholder engagement.

Initial tests of Optimizer WSS comprising MOGA, MCDA and Solution Presentation components have shown the potential of the tool, as part of a suite of tools, to support decisions on water system planning and operations. The paper presents the Optimizer WSS modelling process, the multi-objective model formulation used for testing, and the initial test results under a hypothetical streamflow and demand scenario.

Keywords: Multi-objective optimisation, genetic algorithms, multi-criteria decision analysis, water supply, REALM 
Kularathna et al., Multi-Objective optimisation using Optimizer WSS to support operation and planning decisions of Melbourne water supply system

\section{INTRODUCTION}

The past decade has been a very challenging drought period for many water supply systems in Australia. Water authorities have responded with various demand and supply measures ranging from water conservation and water restrictions to alternative non-traditional supply sources. In the case of Melbourne's water supply system, the supply measures included major water infrastructure projects including an interbasin pipeline and a seawater desalination plant. Many of the above measures affect the key objectives of water utilities such as providing a reliable supply of low cost, high quality water while maintaining environmental health. Optimisation of the water supply system considering the above objectives is a key goal of Melbourne Water and its stakeholders. This optimisation involves identifying the right mix of decisions on (1) key operations and (2) future supply-demand balance.

The above optimisation is complex due to: the need for a long-term oriented solution, uncertain future streamflow conditions, complexity of the water supply system, the need to best meet the multiple needs and objectives of all stakeholders, and the large array of supply and demand measures available for the long-term. In the case of water supply systems that have large storage capacities to store water for use over several years, any decisions on supply and demand measures must also be based on a trade-off of short-term operation decisions such as greater use of higher cost sources versus longer-term supply and demand measures. This is a particular consideration for Melbourne's system which can take many years to fill, causing the impacts of any decisions on operations or supply-demand balance to be carried forward in the systems 'memory' for many years into the future, if not several decades.

Despite the need for optimisation, and many past research efforts, specific tools are not widely available or used in water resources management, particularly for large-scale problems that are characterised by uncertainty. Labadie (2004) reviewed the past efforts in the above area. There had been many other research efforts, over several decades, on improving the applicability of traditional optimisation methodologies to real water supply systems including Kularathna (1992), Draper (2001) and Castelletti et al. (2007). Despite these research efforts, the continued lack of popularity of these methods has been partly due to their drawbacks including the complexity, the need for a simplified representation of the system, lack of flexibility in model formulation, lack of consideration for multiple objectives and multiple stakeholders, and the expertise required. Despite the lack of wide availability or application of traditional optimisation tools for large-scale water resources management problems, Nicklow et al. (2010) indicate that there has been an increase during the last two decades in the development and application of various types of evolutionary algorithms such as Genetic Algorithms (GA). These have been mainly in the areas of water distribution systems, water and wastewater treatment, hydrologic modeling, groundwater systems and parameter identification. Some of the recent studies that have had similarities to the current study in terms of the problem addressed or methodology used are as follows: Cui and Kuczera (2005) assessed the ability of GA to optimise key operating decisions for a complex urban water supply system with nine reservoirs and interbasin transfers subject to a highly variable climate. The study showed that the GA produced results that were consistent with the strategy that minimised total expected costs. Bekele and Nicklow (2005) demonstrated the effectiveness of an integrative modeling framework that combined a watershed model with a multi-objective evolutionary algorithm to explore the tradeoff of ecosystem services and agricultural commodity production. Pianosi and Galelli (2010) presented an approach that combined artificial neural networks and multi-objective genetic algorithms to optimise decision rules for operating a multi-purpose system of three interconnected reservoirs.

In contrast to optimisation tools, simulation modelling tools, such as REALM (Perera et al., 2005), have been widely used by many water authorities in Australia to assist with system planning and operations. REALM simulates the water allocation within a system in accordance with user-defined operating rules, but does not optimise the system operations over time. In the past, the reliance on user-defined operating rules has been satisfactory, as these rules reflected past system operations that have been improved gradually to an 'optimal' level. More recently, the introduction of non-traditional supply sources for which no past experience is available and the increased focus on multiple objectives have highlighted the shortcomings of a simulation modeling approach.

In response to the above, Melbourne Water engaged water system optimisation specialists Optimatics to develop a decision support system, Optimizer WSS, comprising multi-objective optimisation and multi-criteria decision analysis. Optimizer WSS employs a Multi-Objective Genetic Algorithm (MOGA) optimisation 'engine' which in turn uses Melbourne Water's existing REALM simulation model. The use of an existing simulation model and a multi-objective optimisation means that this approach addresses the drawbacks of traditional optimisation approaches outlined previously. The paper presents the findings from an initial test application of Optimizer WSS at Melbourne Water to support decisions on key operations and supply measures. The optimisation problem addressed by Optimizer WSS is one in which selected key 
Kularathna et al., Multi-Objective optimisation using Optimizer WSS to support operation and planning decisions of Melbourne water supply system

operating decisions and supply measures are optimised over a long-term planning horizon of up to 30 years. Optimizer WSS does not address the detailed and interactive optimisation of short-term operations for annual operation planning purposes, which is addressed in Kularathna et al. (2011). However there are linkages between the two optimisation approaches, as Optimizer WSS can provide key inputs to the short-term optimisation.

The paper is structured as follows. Section 2 provides a description of the Melbourne water supply system. Section 3 outlines the optimisation model formulation. The modeling process is described in Section 4. Section 5 outlines the findings from an example application, followed by summary and conclusions.

\section{MELBOURNE WATER SUPPLY SYSTEM}

Melbourne Water is a Victorian government owned company that provides wholesale water supply and sewerage services to Melbourne's retail water companies, and manages rivers and creeks, floodplains and the regional drainage system. In addition, Melbourne Water provides limited water supplies to several regional water authorities. The water supply system managed by Melbourne Water is a complex interconnected system of 10 storage reservoirs, over 40 service reservoirs, 160,000 hectares of catchments and a transfer system comprising hundreds of kilometres of pipelines, tunnels and aqueducts. The transfer system links Melbourne's storage reservoirs with the areas serviced by the three retail water companies in Melbourne and regional water authorities. Figure 1 shows the storage reservoirs and wholesale water supply system managed by Melbourne Water, including the planned supply from the Victorian seawater desalination plant.

Melbourne's storage reservoirs have a combined storage capacity of 1,810GL. The average volume of water supplied from the system for urban use over the last 5 years was about 390GL per year. The current water harvesting and transfer system comprises gravity fed supplies from protected catchments as well as pumped and fully treated supplies from unprotected catchments. The existing and new supply sources that require water treatment and pumping are associated with higher costs. Some of these sources such as the desalinated supply can be unaffected by climate.

Streamflow into Melbourne's water supply is impacted by relatively high climate variability. Despite the large storage capacity of the system, prolonged drought periods in the past have caused significant drawdown of system storage levels over a number of years, resulting in water restrictions.

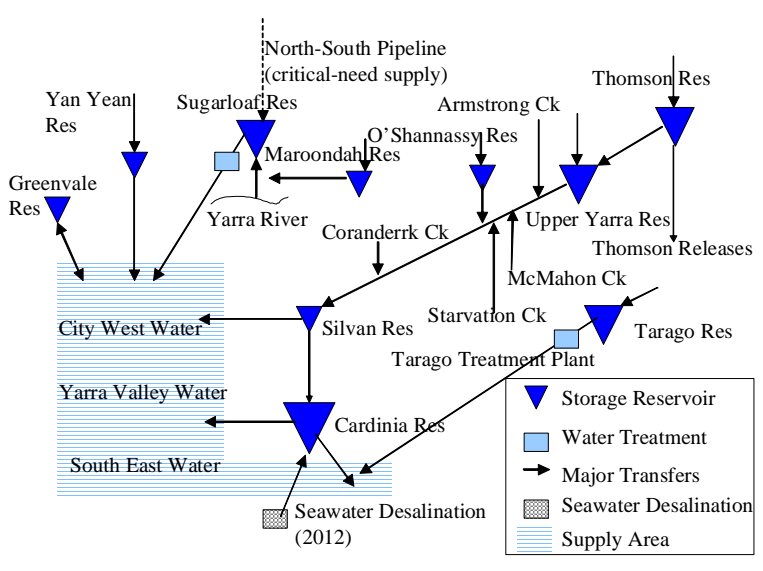

Figure 1. Melbourne water supply system.

\section{OPTIMISATION PROBLEM AND MODEL FORMULATION}

The optimisation problem addressed by Optimizer WSS is the identification of optimal solutions for key operating decisions and supply measures for the water supply headworks system that comprises water harvesting sites, storage reservoirs and bulk transfer paths. The proposed optimisation process takes into account four key considerations: (1) multiple objectives (2) long-term view of up to 30 years (3) uncertain future climate, and (4) multiple stakeholders.

\subsection{Multi-objective optimisation model formulation}

An optimisation model formulation comprises three key components; (1) objective functions that define the aims of optimisation, (2) decisions that can be varied to achieve the objectives, and (3) constraints that indicate the limitations imposed on the optimisation.

As indicated previously, Optimizer WSS uses Melbourne Water's REALM simulation model to assess the consequences of millions of alternative decisions, in an efficient search process that identifies improved solutions as the search progresses. The broad roles of Optimizer WSS are to (1) alter the decisions within the REALM model, (2) run the REALM model for the 30-year optimisation time horizon, (3) read the modelled outputs of REALM, (4) compare the modelled system behaviour to those modelled in previous alterations and (5) identify the next set of decision alterations. The above 5 step process is then repeated until the solutions converge to a desired level of incremental improvement. The alterations are carried out in a manner 
Kularathna et al., Multi-Objective optimisation using Optimizer WSS to support operation and planning decisions of Melbourne water supply system

ensuring the search for the 'optimal' solution evolves as the search progresses. The evolutionary search methodology used in Optimizer WSS is the Multi-Objective Genetic Algorithm approach known as NSGA-II (Deb et al., 2002).

As the above process relies on system behaviour modelled by REALM, any decisions, objective functions or constraints that can be represented within REALM can be incorporated into the optimisation process. Optimizer WSS provides the flexibility to include additional objective functions and constraints outside REALM as well, if required. The objective functions used in this study are:

- Minimise total discounted cost in operating and upgrading the system,

- Maximise the additional water released to the environment above the required release volumes, and

- Maximise security of supply (achieved by minimising economic losses to consumers caused by demand reductions).

The decisions of the optimisation problem belonged to four categories (1) volumes of water to be taken from various sources that have differential costs, (2) the timing of these intakes, (3) the distribution of water within the water supply system, and (4) the timing of future water supply augmentations.

The constraints of the optimisation problem included the minimum environmental flow requirements and water supply system asset capacity limitations (e.g. reservoir storage capacities and pipeline capacities). Most of these constraints were included within the REALM model.

The four key considerations outlined previously and the complexity of the water supply system result in an extensive optimisation problem. An example of the extent of the optimisation problem can be provided in reference to a 30-year optimisation problem formulated in monthly time steps. Assuming 10 operating decisions are needed for each month and each decision involves choosing from 5 discrete options, the number of possible combinations of decisions in the optimisation problem that considers a 30-year time frame would be $5^{3600}$. Although most of these decision combinations may be infeasible, even a small fraction of feasible decisions among these combinations still presents a huge decision problem. The problem size further increases when considering the added complexities of uncertain climate and future supply demand options, confirming the need for a model-assisted optimisation process.

\section{OPTIMISATION PROCESS}

The proposed process enables handling the optimisation problem through two stages of modeling and decision analysis involving a high level of stakeholder participation. Stage 1 of the process involves 'screening' of solutions using multi-objective optimisation. The screened solutions are assessed using multi-criteria decision analysis to narrow down the better solutions further. In Stage 2, the better solutions are assessed for their robustness under alternative streamflow scenarios. The results of this assessment are presented to stakeholders to assist in selecting the most robust solution through a second multi-criteria decision analysis.

\subsection{Stage 1: Screening of solutions}

The Stage 1 of the optimisation process is detailed below with reference to Figure 2.

Define Modelling Scenarios: The first step of the process includes identifying a set of streamflow and demand scenarios that encompasses the range of possibilities over the optimisation time horizon.

Multi-objective Optimisation: In this step, Optimizer WSS is run to generate ('screen') optimal solutions. The optimisation can be carried out through two alternative ways involving (1) simultaneous optimisation across a range of scenarios, or (2) one-at-a-time optimisation for several streamflow scenarios. The process covered in this paper is based on the latter. A different optmisation formulation and process has been tested for simultaneous optimisation across scenarios, which is outside the scope of this paper.

Pareto-Optimal Solutions: Unlike a single objective optimisation which only produces a single optimal solution

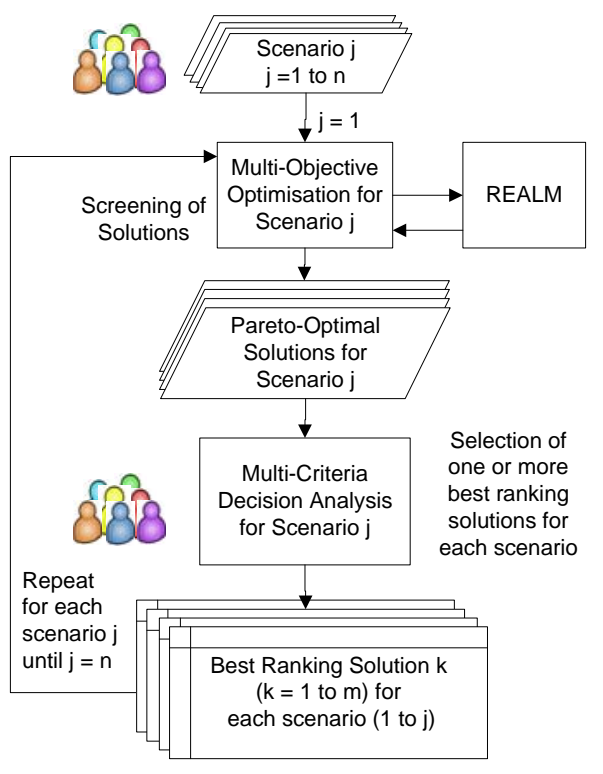

Figure 2. Stage 1 of the optimisation process. 
Kularathna et al., Multi-Objective optimisation using Optimizer WSS to support operation and planning decisions of Melbourne water supply system

in terms of the objective function value, a multi-objective optimisation identifies multiple optimal solutions comprising those that are best for each individual objective as well as many solutions that trade off the objectives to different extents. Figure 3 shows an example of these solutions, known as Pareto-Optimal solutions (or Pareto Front), for two competing objectives: operating cost and water supply security. As indicated by Figure 3, the solutions retained as Pareto-Optimal solutions are those that cannot be further improved in terms of any one objective without the worsening of another objective. All other solutions are screened-out by the multi-objective optimisation. An important aspect to note is that a 'solution' indicated by a point on Figure 3

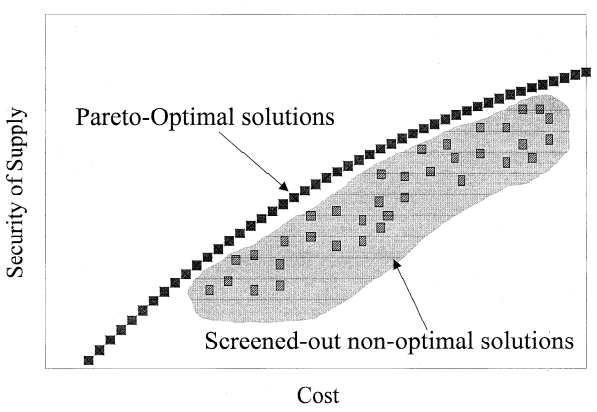

Figure 3. Pareto-Optimal Solution Example. represents a series of decisions identified by Optimizer WSS on key system operations and supply measures over the optimisation time horizon.

Multi-Criteria Decision Analysis: The Pareto-Optimal solutions represent a set of equally good solutions from which the selection of one solution involves a trade-off of one or more other objectives. This tradeoff needs to be based on the preferences of stakeholders, and is assisted by a Multi-Criteria Decision Analysis (MCDA) which enables ranking of the Pareto-Optimal solutions. The criteria used in the MCDA can include the objectives used in the optimisation as well as many additional assessment criteria that may not have been used as objectives. For the purpose of MCDA, the Pareto-Optimal solutions can be visualised in a table in which each row represents an individual solution and each column represents an assessment criterion.

The MCDA approach adapted for Optimizer WSS is the well known weighted-sum method which relies on identifying the relative importance to stakeholders of various criteria, expressed as 'criteria weight factors'. However, obtaining this information from stakeholders is not a straightforward task, particularly when there are more than a couple of criteria. To assist identifying the criteria weights, the method of pair-wise comparison of criteria by stakeholders has been adapted. A description of the pair-wise comparison approach can be found in Fleming (1999). The stakeholder inputs obtained through this method are used to derive several different sets of criteria weights representing those of individual stakeholders and the averaged criteria weights of all stakeholders. When the solutions are ranked using the different sets of criteria weights, several different 'best-ranking' solutions can be identified for the same streamflow/demand scenario, with one solution for each set of criteria weights. The process shown in Figure 2 is then repeated for different streamflow/demand scenarios to identify a range of best-ranking solutions, comprising several such solutions for each streamflow/demand scenario.

\subsection{Stage 2: Selecting the most robust solution}

The aim of this step is to identify from the range of bestranking solutions a solution that is the most robust under a range of future streamflow scenarios. As shown in Figure 4, REALM simulation is carried out to assess the system behaviour resulting from each individual best-ranking solution (which comprises a series of key operating decisions and supply measures) under a range of alternative streamflow scenarios. The system behaviours simulated by each solution can then be summarised by a set of assessment criteria. These assessment criteria may correspond to those used in the Stage-1 MCDA, but need to be expressed differently to summarise the wide range of system performances under alternative streamflow scenarios. As an example, the criterion 'total discounted cost' used in the Stage-1 MCDA may need to be represented in the current step through one or more criteria such as total expected discounted cost, minimum discounted cost and the maximum discounted cost. Alternatively, several discounted cost estimates may be used, each of which corresponding to a different probability of exceedance. The criteria values of various solutions are subsequently assessed in a second 
Kularathna et al., Multi-Objective optimisation using Optimizer WSS to support operation and planning decisions of Melbourne water supply system

MCDA to identify the recommended best-ranking solution(s). As with the Stage-1 MCDA, this analysis can assist in selecting several recommended solutions if the stakeholder inputs are considered individually, or one solution if the stakeholder inputs are combined. The recommended solutions can demonstrate to the stakeholders the implications of their preferences and provide valuable information to assist identifying a mutually acceptable solution.

\section{SOLUTIONS FROM AN EXAMPLE APPLICATION}

The example solutions presented below are from the outputs obtained from Stage 1 of the optimisation process using one streamflow and demand scenario. The solutions presented are intended for illustration only and do not indicate the potential behaviour of Melbourne Water supply system. Description of the assumptions used in the example has been intentionally omitted from this paper to reinforce the above intent.

The optimisation problem as formulated in Section 3.1 was run in Optimatics' cloud computing cluster using 120 computing cores. The run was completed in 6 hours and produced more than 650 'screened' or ParetoOptimal solutions, each of which comprises an equally good set of key operating decisions and supply measures for the 30 years ahead. Figure 5 summarises the objective function values of the above solutions. These solutions were ranked through MCDA to select several best-ranking solutions for further analysis. The assessment criteria used in the MCDA included the three objective functions used in the optimisation as well as additional criteria that describe other aspects of the solutions. The additional criteria included the discounted cost over the first 5 years, and, the storage volume at the end of 5 years.

The MCDA was carried out with four different sets of criteria weights. These corresponded to the individual preferences of three hypothetical stakeholders and the combination of the three preferences. The respective preferences of three

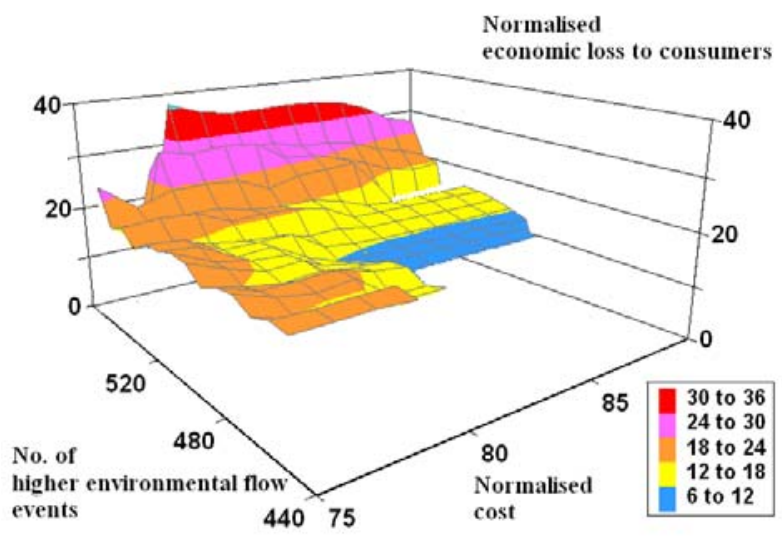

Figure 5. Pareto-Optimal solutions. stakeholders were: (1) highest preference for minimising long-term cost, (2) highest preference for maximising long-term security of supply, and (3) equally high preferences for minimising long-term cost and maximising long-term security of supply. The fourth set of weights was based on the combined weights of the three stakeholders that were biased towards higher security of supply. The objective function on maximising the additional water released to the environment was not assigned the highest preference of any hypothetical stakeholder, due to the simplified way in which potential environmental benefits are currently represented in that particular objective function. Table 1 summarises the four selected solutions.

Table 1 shows that the Solutions 1 and 2 satisfy the respective preferences of the two stakeholders. In terms of Figure 3, these two solutions can be described as solutions closer to the two respective ends of the Pareto Front. In Figure 5 which has an additional dimension of a third objective, these two 'ends' are represented by respective areas on the Pareto Front with low cost and low economic loss to consumers. The weights of the third stakeholder indicate equal preferences for the above two objectives. Hence the resulting best-ranking solution satisfies both objectives to some extent but is not the best in terms of any of the two objectives. In terms of Figure 3, this solution is from an intermediate point on the Pareto Front. Figure 5 confirms the intermediate nature of the solution in regards to the above two objectives. However this third solution has a better environmental flow performance. Finally, the combined weights of all three stakeholders are biased towards security of supply and the resulting best ranking solution is similar to Solution 2.

The above solutions demonstrate the use of the optimisation process to assist in selecting different optimal solutions that

Table 1. Summary of selected solutions.

\begin{tabular}{|l|c|c|c|c|}
\hline \multicolumn{1}{|c|}{ Criterion } & Solution 1 & Solution 2 & Solution 3 & Solution 4 \\
\hline & $\begin{array}{c}\text { Sakeholder 1 } \\
\text { Preference } \\
\text { Priority: } \\
\text { Lower Cost }\end{array}$ & $\begin{array}{c}\text { Stakeholder 2 } \\
\text { Preference } \\
\text { Priority: } \\
\text { Higher } \\
\text { Security of } \\
\text { Supply }\end{array}$ & $\begin{array}{c}\text { Stakeholder 3 } \\
\text { Preference } \\
\text { Priority: } \\
\text { Equal }\end{array}$ & $\begin{array}{c}\text { Combined } \\
\text { Preferences } \\
\text { Priority: } \\
\text { Higher } \\
\text { Security of } \\
\text { Supply }\end{array}$ \\
\hline Discounted total cost* over 30 years & 76 & 87 & 79 & 87 \\
\hline No. of events with higher environmental flows over 30 years & 472 & 510 & 522 & 509 \\
\hline Economic losses* to consumers over 30 years & 16 & 10 & 12 & 10 \\
\hline 5-year discounted total cost* & 11 & 24 & 14 & 24 \\
\hline 5-year End storage volume (\% full) & $45 \%$ & $49 \%$ & $47 \%$ & $49 \%$ \\
\hline *Costs and economic losses shown are normalised between 0 and 100 & & &
\end{tabular}


Kularathna et al., Multi-Objective optimisation using Optimizer WSS to support operation and planning decisions of Melbourne water supply system

(1) achieve each of the individual objectives best (2) trade-off different objectives to different extents, and (3) represent the combined preferences of all stakeholders. The limited number of solutions selected through this approach can subsequenlty be assessed in Stage 2 of the optimisation process to identify the most robust solution(s) that would in turn assist in identifying a solution acceptable to all stakeholders.

\section{SUMMARY AND CONCLUSIONS}

The paper presented an initial test application of a multi-objective optimisation approach for optimising key operating decisions and supply measures for a complex water supply system. The optimisation approach provides many advantages over traditional optimisation methodologies. They include the flexible multiobjective optimisation formulation configured within an existing simulation model, long-term view, the high level of stakeholder participation in the process, the high level of detail modelled, and consequently the wide range of information that can be supplied to the stakeholders. The process described in this paper relies on a scenario based optimisation to identify a robust solution. An alternative optimisation formulation has also been developed that is able to optimise across a range of scenarios. The preliminary test application presented in this paper indicates the potential of Optimizer WSS, as part of a suite of tools, in optimising the key operating decisions and supply measures for the Melbourne Water supply system. The methodology is also applicable to other water supply systems, but the key challenges in applying the methodology include: (1) appropriately formulating the optimisation problem to capture key drivers of system behaviour and minimise the size of the problem to practical levels, (2) stakeholder engagement in the application of a comprehensive modelling and decision support process, and (3) encompassing the growing boundaries of 'water systems' to achieve whole-of-water-cycle optimisation.

\section{ACKNOWLEDGMENTS}

The feasibility study preceding this project was funded by a research grant provided by the Victorian Department of Innovation, Industry and Regional Development through its Market Validation Program.

\section{REFERENCES}

Bekele, E.G., and J.W. Nicklow (2005). Multiobjective management of ecosystem services by integrative watershed modelling and evolutionary algorithms. Water Resources Research, 41(W10406).

Castelletti, A., D. de Rigo, A.E. Rizzoli, R. Soncini-Sessa, and E. Weber (2007). Neuro-dynamic programming for designing water reservoir network management policies. Control Engineering Practice, 15(8), 1031-1038.

Cui, L., and G. Kuczera (2005). Optimizing water supply headworks operating rules under stochastic inputs: Assessment of genetic algorithm performance. Water Resources Research, 41(W05016).

Deb, K., A. Pratap, S. Agarwal, and T. Meyarivan (2002). A fast and elitist Multiobjective Genetic Algorithm: NSGA-II. IEEE Transactions on Evolutionary Computation, 6(2), 182-197.

Draper, A.J. (2001). Implicit stochastic optimization with limited foresight for reservoir systems. Ph.D. thesis, University of California, Davis.

Fleming, N. (1999). Sustainability and water resources management for the Northern Adelaide Plains, South Australia. Ph.D. thesis, University of Adelaide, Adelaide, Australia.

Kularathna, M.D.U.P. (1992). Application of dynamic programming for the analysis of complex water resources systems: A case study on the Mahaweli river basin development in Sri Lanka. Ph.D. thesis, Wageningen Agricultural University, The Netherlands.

Kularathna, M.D.U.P., B. Baker, and S. Ennor (2011). Interactive optimisation modelling using OPTIMISIR to support Melbourne water supply system operations. Submitted to MODSIM 2011 International Congress on Modelling and Simulation, Perth, 12-16 December.

Labadie, J. (2004). Optimal operation of multireservoir systems: State-of-the-art review. Journal of Water Resources Planning and Management, 130(2), 93-111.

Nicklow, J.W., P. Reed, D. Savic, T. Dessalegne, L.Harrell, A. Chan-Hilton, M. Karamouz, B. Minsker, A. Ostfeld, A. Singh, and E. Zechman (2010). State of the art of genetic algorithms and beyond in water resources planning and management. Journal of Water Resources Planning and Management, 136(4), 412-432.

Perera, B.J.C., B. James, and M.D.U. Kularathna (2005). Computer software tool REALM for sustainable water allocation and management. Journal of Environmental Management, Elsevier, 77, 291-300.

Pianosi, F., and S. Galelli (2010). Neural network and evolutionary algorithms for water resources management. Proceedings of the 9the International Conference on Hydroinformatics, Tianjin, China, 7-11 September. 